\title{
La investigación sobre Internet en las facultades de Comunicación españolas. Análisis bibliométrico de tesis doctorales (1997-2012)
}

\author{
Jesús DÍAZ-CAMPO \\ jesus.diaz@unir.net \\ Profesor asociado \\ Facultad de Empresa y Comunicación \\ Universidad Internacional de La Rioja (UNIR)
}

Recibido: $10 / 02 / 2014$

Aceptado: 12/03/2014

\section{RESUMEN}

Esta investigación analiza las tesis doctorales sobre Internet defendidas en las facultades de Comunicación españolas en el periodo 1997-2012. La base de datos utilizada para localizar las tesis ha sido Teseo. El número de tesis analizadas en el periodo estudiado asciende a 167 títulos. La metodología empleada es el análisis bibliométrico. Se analiza la producción por años, idioma de lectura, sexo del doctorando, universidades y facultades de lectura, directores y miembros de los tribunales evaluadores. Los resultados muestran ciertos altibajos en la producción de tesis, si bien la tendencia creciente es constante en los últimos años analizados. Asimismo, el español es con diferencia el idioma más utilizado por los autores, entre los cuales hay ligera mayoría de hombres frente a mujeres. La Complutense es la universidad más prolífica, si bien el director con mayor producción pertenece a la Universidad de Murcia. Por el contrario, los académicos más solicitados para formar parte de los tribunales sí que provienen sobre todo de la Complutense. Estos datos se comparan con los obtenidos en otros análisis bibliométricos recientes y también relacionados con el ámbito de la Comunicación, como los referidos a la Publicidad (Marcos Recio, Martínez Pestaña y Blasco López, 2012; Martínez Pestaña, 2011a y 2011b); Televisión (Repiso, Torres y Delgado, 2011); Radio (Repiso, Torres y Delgado, 2012); y Cine (Repiso, Delgado y Torres, 2012).

Palabras clave: Tesis doctorales, Internet, Comunicación, bibliometría, universidad, España

\section{Internet research in Spanish communication faculties. Bibliometric analysis of doctoral dissertations (1997-2012)}

\begin{abstract}
This study analyses the production of doctoral dissertations on the Internet which have been defended in Communication departments in Spain over the period 1997-2012. The TESEO database has been used to search the data. The total number of dissertations produced during the studied period was 167 . Bibliometric analysis techniques have been used in order to analyze the data. Production is analyzed for time period, theses language, $\mathrm{PhD}$ candidate genre, universities and faculties, theses advisors an examination board members. Results show some ups and downs in the production of theses, although the trend is growing steadily in recent years. Also, Spanish is by far the most popular language used by the authors, and there are slightly more men than women among them. Complutense University is the most prolific one, although the most prolific director belongs to the University of Murcia. However, most sought professors to be part of the examination boards come mainly from the Complutense University. These data are compared with those obtained in other recent bibliometric analysis and related to the field of communication, such as those on Advertising (Marcos Recio,
\end{abstract}


Martínez Pestaña y Blasco López, 2012; Martínez Pestaña, 2011a y 2011b); Television (Repiso, Torres y Delgado, 2011); Radio (Repiso, Torres y Delgado, 2012); and Cinema (Repiso, Delgado y Torres, 2012).

Keywords: Doctoral theses, Internet, communication, bibliometric studies, university, Spain

\section{INTRODUCCIÓN}

La aparición de Internet y su consolidación en la década de los 90 constituye uno de los acontecimientos más importantes en el devenir de los medios de comunicación. El surgimiento de la red ha transformado la propia naturaleza del proceso comunicativo, ha obligado a los medios ya existentes a adaptarse a la nueva realidad, y ha dado lugar a la creación de otros nuevos.

Pues bien, aunque los análisis sobre Internet y medios de comunicación han proliferado en los últimos años, este trabajo pretende abordar la cuestión desde una perspectiva diferente: una de las vías para caracterizar la investigación que se está realizando en un campo concreto son las tesis doctorales, cuya lectura sirve para certificar la capacidad investigadora de quienes la llevan a cabo (Casanueva y Caro, 2013). A través de estos documentos se pueden analizar las diversas líneas de investigación, sus rasgos más característicos, su evolución y su grado de consolidación.

En ese sentido, una tesis doctoral supone el primer gran trabajo científico que realiza un investigador después de un, por lo general, largo proceso de aprendizaje y marca en muchos casos la línea o al menos una de las líneas de investigación que su autor seguirá en el futuro, empezando por el hecho de que tras su defensa es muy probable que la intente difundir a través de artículos y comunicaciones.

Por ello, el objetivo principal de esta investigación es recopilar y analizar los datos estadísticos fundamentales relativos a la producción de tesis doctorales sobre Internet defendidas en los departamentos de Periodismo, Publicidad y Comunicación Audiovisual de las universidades españolas. La herramienta metodológica empleada es el análisis bibliométrico, es decir, la aplicación de técnicas cuantitativas para estudiar las características bibliográficas de dichas tesis doctorales.

En ese sentido, cuando se ha cumplido ya la veintena de años desde la consolidación de Internet como nuevo medio de comunicación, consideramos ha pasado ya un periodo de tiempo suficiente para comenzar a caracterizar su investigación en todo este periodo, a través del estudio de las tesis doctorales.

En España se han publicado numerosos análisis bibliométricos sobre tesis doctorales, la mayoría de los cuales se recogen en el estudio de Fuentes y Arguimbau (2010), que distingue hasta 18 áreas temáticas diferentes cuya producción de tesis doctorales ha sido analizada y caracterizada. De entre todos ellos, en el campo de la Comunicación, pueden citarse los estudios sobre Publicidad (Martínez Pestaña, 2004) y sobre Relaciones Públicas (Castillo y Xifra, 2006; Xifra y Castillo, 2006). 
Con posterioridad al estudio de Fuentes y Arguimbau, han aparecido otros análisis también enmarcados en el área de la Comunicación, como los dedicados a la Publicidad (Marcos Recio, Martínez Pestaña y Blasco López, 2012; Martínez Pestaña, 2011a y 2011b); la Televisión (Repiso, Torres y Delgado, 2011); la Radio (Repiso, Torres y Delgado, 2012); y el Cine (Repiso, Delgado y Torres, 2012; Repiso, Torres-Salina y Delgado López-Cózar, 2013).

Del mismo modo, no se puede pasar por alto la figura de Daniel Jones, quien llevó a cabo una intensísima tarea para medir y caracterizar la investigación en Comunicación realizada en España, que se materializó en diversos trabajos, entre los que destaca su análisis de las tesis doctorales sobre comunicación defendidas en España entre 1926 y 1998 (Jones et al, 2000), que ha servido de base para un gran número de investigaciones posteriores.

Los trabajos de este tipo realizados en España se completan con los estudios similares pero centrados en una comunidad autónoma o en un ámbito geográfico más concreto. Es el caso de las investigaciones de Jones y Baró sobre Cataluña (1997); Landa Montenegro sobre el País Vasco (2004); y Arboledas Márquez y Herrero Solana referido a las universidades de Sevilla y Málaga (2013).

La presencia de Internet como línea de investigación en estos trabajos es minoritaria. Así, Arboledas Márquez y Herrero Solana (2013) localizan tres descriptores relacionados con las nuevas tecnologías (Tecnología de las Comunicaciones, Internet e Intranet, y Ciencias Tecnológicas). Cada uno de ellos aparece en dos ocasiones en la relación de tesis doctorales leídas en la Universidad de Málaga. Mientras, en los trabajos defendidos en la Universidad de Sevilla no existe ningún descriptor relacionado con las nuevas tecnologías que haya sido localizado en un mínimo de dos ocasiones.

Por su parte, Landa Montenegro (2004) menciona en su análisis sobre las tesis doctorales leídas en el País Vasco un total de 14 tesis doctorales (de un total de 152 analizadas) relacionadas con "Nuevos Medios" en su categorización en función del medio de comunicación analizado.

Finalmente, ni el trabajo de Jones y Baró (2000) sobre Cataluña, ni el de Jones et al (2000) recogen Internet o las nuevas tecnologías en sus respectivas clasificaciones según el medio de comunicación estudiado.

\section{METODOLOGÍA}

La fuente de información principal para la realización de esta investigación ha sido la base de datos bibliográfica TESEO, dependiente del Ministerio de Educación, Cultura y Deporte, accesible de forma gratuita a través de Internet y que proporciona información sobre las tesis doctorales leídas en España desde 1976.

Como señalan diversos estudios (Fuentes y Arguimbau, 2010; Martínez Pestaña, 2004, 2011a y 2011b; Repiso, Delgado y Torres, 2004; Repiso, Torres y Delgado, 2011 y 2012), TESEO no es ni mucho menos una base de datos 
exhaustiva, y es imposible garantizar que todas las bases de datos leídas hayan sido registradas, pues su fiabilidad depende, entre otros factores, del rigor y la precisión con la que los doctorandos y los tribunales cumplimentan los diversos formularios. Sin embargo, lo cierto es que puede considerarse como el recurso más completo y de obligada consulta para cualquier trabajo relacionado con las tesis doctorales de las universidades españolas (Sorli y Merlo, 2002: 203).

Asimismo, para llevar a cabo la presente investigación, se ha llevado a cabo un análisis previo similar al puesto en práctica por Fuentes y Arguimbau (2010): comparar los datos recogidos por el Instituto Nacional de Estadística (INE) y por TESEO correspondientes al número total de tesis doctorales leídas en España en el periodo analizado, exceptuando el curso 2011-2012, cuyos datos no recoge el INE en el momento de la realización de este trabajo.

Como puede verse (Tabla 1) el grado de exhaustividad de TESEO es bastante alto, a la vez que significativamente superior al obtenido por ejemplo por Fuentes y Arguimbau (2010), quienes comprobaron que el porcentaje de tesis doctorales leídas en España que recopilaba TESEO alcanzaba el 87,3\%.

En este caso ese porcentaje global alcanza el 92,5\%, al tiempo que resulta muy significativo el hecho de que el porcentaje sea incluso superior a la media en los tres últimos cursos académicos que comprende el periodo analizado por la presente investigación.

Este dato constituye una gran diferencia respecto a otros trabajos precedentes, como el de Repiso, Torres y Delgado (2011), que a pesar de haber recogido los datos en 2010, únicamente analizo las tesis doctorales leídas hasta 2007, ya que el porcentaje de cobertura de Teseo no llegaba en algún caso ni siquiera al 70\%. En este sentido, los datos obtenidos por nuestra investigación apuntan a una agilización en el proceso de envío y registro de las fichas de las tesis. Por ello, a diferencia de algunos de los trabajos anteriormente mencionados, se ha considerado adecuado extender el periodo analizado hasta 2012. 


\begin{tabular}{|l|c|c|c|c|}
\hline Curso & Tesis INE & Tesis TESEO & Diferencia & Porcentaje \\
\hline $\mathbf{1 9 9 7 - 1 9 9 8}$ & 5.931 & 5.850 & 81 & $98,6 \%$ \\
\hline $\mathbf{1 9 9 8 - 1 9 9 9}$ & 5.984 & 5.193 & 791 & $86,8 \%$ \\
\hline $\mathbf{1 9 9 9 - 2 0 0 0}$ & 6.408 & 6.109 & 299 & $95,3 \%$ \\
\hline $\mathbf{2 0 0 0 - 2 0 0 1}$ & 6.380 & 5.913 & 467 & $92,7 \%$ \\
\hline $\mathbf{2 0 0 1 - 2 0 0 2}$ & 6.936 & 6.418 & 518 & $92,5 \%$ \\
\hline $\mathbf{2 0 0 2 - 2 0 0 3}$ & 7.467 & 7.003 & 464 & $93,8 \%$ \\
\hline $\mathbf{2 0 0 3 - 2 0 0 4}$ & 8.176 & 7.259 & 917 & $88,8 \%$ \\
\hline $\mathbf{2 0 0 4 - 2 0 0 5}$ & 6.902 & 6.349 & 553 & $91,9 \%$ \\
\hline $\mathbf{2 0 0 5 - 2 0 0 6}$ & 7.159 & 6.315 & 844 & $88,2 \%$ \\
\hline $\mathbf{2 0 0 6 - 2 0 0 7}$ & 7.150 & 6.380 & 770 & $89,2 \%$ \\
\hline $\mathbf{2 0 0 7 - 2 0 0 8}$ & 7.302 & 6.646 & 656 & $91,0 \%$ \\
\hline $\mathbf{2 0 0 8 - 2 0 0 9}$ & 7.915 & 7.538 & 377 & $95,2 \%$ \\
\hline $\mathbf{2 0 0 9 - 2 0 1 0}$ & 8.596 & 8.227 & 369 & $95,7 \%$ \\
\hline $\mathbf{2 0 1 0 - 2 0 1 1}$ & 8.915 & 8.443 & 472 & $94,7 \%$ \\
\hline Total & 101.221 & 93.643 & 7.578 & $92,5 \%$ \\
\hline
\end{tabular}

Tabla 1. Comparativa de las tesis registradas por INE y TESEO (España, 19982007)

No obstante, buscando alcanzar unos resultados lo más exhaustivos posibles, la estrategia seguida ha sido similar a la empleada en varios trabajos anteriores de este tipo (Repiso, Delgado y Torres, 2004; Repiso, Torres y Delgado, 2011 y 2012; Casanueva y Caro, 2013), es decir, realizar una búsqueda exhaustiva de las tesis correspondientes a los departamentos de periodismo, comunicación audiovisual y publicidad de todas las universidades españolas. A continuación se analizaron las frecuencias terminológicas en los títulos de los resultados obtenidos y, con los términos más frecuentes, se realizó otra búsqueda en los campos "Título" y "Resumen".

El criterio de selección de las tesis a partir de las bases de datos, basado en principio en parámetros estrictamente lingüísticos, dio paso después a elementos más analíticos. De este modo, se seleccionaron tesis cuyo título y/o resumen englobaban términos como "Internet", "digital", "online" o "web".

En un segundo análisis se descartaron aquellos trabajos en los que Internet no era parte de su objeto de estudio y en los que el hecho de que algunos de los términos mencionados aparecieran en el resumen se debía a que, o bien algunas fuentes utilizadas en la investigación se habían obtenido en la Red, o bien parte del proceso metodológico se había desarrollado en Internet mediante, por ejemplo, la publicación de una encuesta en una página web.

Por último, se descartaron aquellas tesis leídas en 2013, al no haber finalizado todavía el año en curso en el momento de la realización de este trabajo. 
Como resultado final de todo el proceso, se recuperaron un total de 167 registros, el primero de los cuales data de 1997.

La siguiente fase consistió en tratar de normalizar los campos de los registros seleccionados, para intentar corregir los errores e inexactitudes encontrados al realizar la búsqueda, errores como los siguientes:

- Ausencia de la información referente a uno o más miembros del tribunal.

- Falta del dato referente al departamento de lectura.

- Inexactitudes en el día y mes de lectura. En un gran número de las tesis registradas por TESEO aparece como fecha por defecto el 1 de enero del año correspondiente, a pesar de que la tesis realmente ha sido defendida en otro día distinto.

Por todo ello, se complementó la búsqueda con la consulta de otras bases de datos como Dialnet ${ }^{1}$, el buscador Mastesis ${ }^{2}$ y las bases de datos específicas de tesis doctorales del resto de bibliotecas pertenecientes a universidades que cuentan con estudios de Comunicación. Esta última fuente sirvió para recuperar el dato del director y del departamento de lectura en todos los casos en los que no constaba. No ocurrió lo mismo con la información referente al tribunal evaluador, un dato muy complicado de recuperar si no se encuentra en la referencia de TESEO.

Como señalan Fuentes y Arguimbau (2010: p. 66), las dimensiones más comunes en este tipo de análisis son los indicadores temáticos (disciplinas y áreas de estudio) y los de productividad (directores, tribunales, años, universidades y centros de lectura).Otras variables que se estudian en los trabajos examinados son el idioma de lectura o el sexo de los doctorandos. Por ello, en la base de datos creada para llevar a cabo la presente investigación se insertó la siguiente información

1. Nombre completo del autor

2. Sexo del autor

3. Título completo de la tesis doctoral

4. Año de lectura

5. Universidad en la que tuvo lugar la lectura

6. Departamento y/o facultad en la que se leyó la tesis

7. Director o directores de la tesis

8. Miembros del tribunal

9. Idioma en el que está escrito

Si bien no se puede asegurar que las 167 tesis identificadas coinciden con el número exacto de tesis sobre Internet defendidas en los departamentos de Comunicación en las universidades españolas entre 1997 y 2013, principalmente por los defectos hallados en la cobertura de las bases de datos, sí que se puede afirmar que las tesis finalmente recuperadas con suficientemente representativas y sirven para comprobar cuál ha sido la tendencia en su producción.

\footnotetext{
${ }^{1}$ http://dialnet.unirioja.es/

${ }^{2}$ www.mastesis.com
} 


\section{RESULTADOS}

\subsection{E de la producción global}

El número total de tesis sobre Internet defendidas en los departamentos de Periodismo, Publicidad y Comunicación Audiovisual de las universidades españolas hasta el año 2012 asciende a 167. La primera de ellas fue la investigación de José Luis Macaya Jorquera Información y comunicacion eficaz en las organizaciones a través del World Wide Web, leída el 22 de enero de 1997 en la Facultad de Ciencias de la Información de la Universidad Complutense de Madrid. A título meramente informativo, se ha añadido una nube de palabras (Figura 2) generada con el programa Wordle ${ }^{\circledR}$ a partir de los términos que aparecen en los títulos de las 167 tesis analizadas. El tamaño menor o mayor en la imagen de cada una de las palabras está directamente relacionado con su frecuencia de aparición

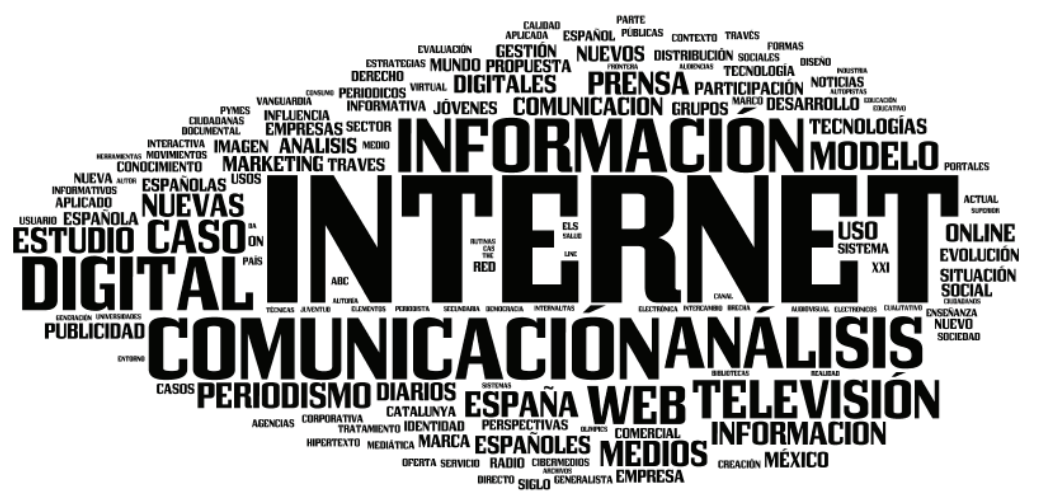

Figura 1. Nube de palabras elaborada con los términos que aparecen en los títulos de las tesis

La tendencia general es al alza (Figura 2) y alcanza su máximo en 2010 con 26 tesis. Sin embargo, ese incremento no es constante a lo largo de todo el periodo analizado, resultando especialmente significativos los datos obtenidos entre $2004 \mathrm{y}$ 2007, que muestran una línea descendente, pasando de 13 tesis en 2007 a 2 en 2004. A partir de ese momento, las cifras experimentan un repunte, que cristaliza en los últimos años, como demuestra el hecho de casi la mitad $(46,1 \%)$ de las tesis se han leído entre 2009 y 2012. 


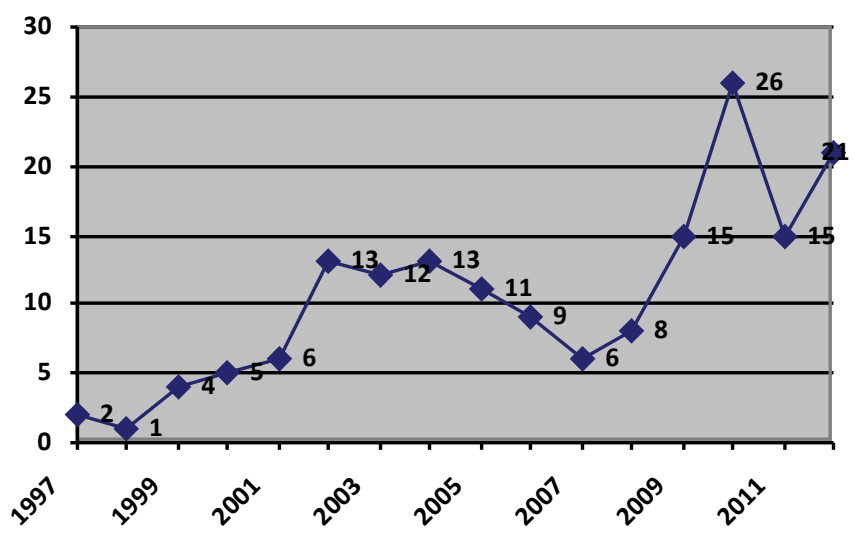

Figura 2. Evolución de la producción de tesis doctorales por años

\section{2. Idioma y sexo de los doctorandos}

El castellano es el idioma empleado por la mayoría de autores de las tesis analizadas, puesto que 158 de ellas $(94,6 \%)$ han sido escritas en esa lengua. El resto de lenguas que tienen carácter oficial en España suman otro 4,4\% que corresponden a cuatro tesis redactadas en catalán y tres escritas en gallego. Por último, en dos de los trabajos analizados $(1,3 \%)$ la lengua utilizada fue el inglés. No se ha localizado ninguna tesis en francés, portugués ni en ninguna otra lengua.

Por lo que respecta al sexo de los doctorandos, los hombres representan el $53,9 \%$ de las autorías, con un total de 90 tesis, mientras que el 46,1\% restante (77 tesis) corresponde a mujeres. Examinando la distribución anual de autorías por sexo, los hombres lideran la producción en la mayoría de años analizados, si bien las cifras obtenidas por las mujeres experimentan una tendencia creciente, hasta llegar a superar a los hombres en 2010 y 2011.

\subsection{Universidades de lectura}

Las tesis localizadas se han defendido en 25 universidades (Tabla 2). La clasificación la lidera la Universidad Complutense de Madrid, en la que se han leído 72 tesis, cerca de la mitad de todas las que se han analizado (43,1\%). Le siguen, a gran distancia la Universidad Autónoma de Barcelona con 16 tesis (9,6\%); la Universidad de Málaga con 10 (5,9\%); la Universidad de Murcia con 9 $(5,4 \%)$; y la Universidad San Pablo-CEU con 7 (4,2\%). Con 6 tesis doctorales leídas se sitúan la Universidad del País Vasco y la Universidad de Sevilla; Mientras, la Universidad de Extremadura, la Universidad de Navarra, y la Universidad de Santiago de Compostela han acogido 5 tesis cada una. En el resto de universidades se han defendido tres o menos tesis doctorales. 
Resultan especialmente llamativas las cifras obtenidas por algunas universidades relativamente "jóvenes" en cuanto al ámbito de la Comunicación se refiere, como la Universidad de Málaga, la de Murcia, la de Sevilla o la de Extremadura.

\begin{tabular}{|l|c|c|}
\hline Universidad & $\begin{array}{c}\mathrm{N}^{\mathrm{o}} \\
\text { tesis }\end{array}$ & $\%$ \\
\hline Complutense Madrid & 72 & $43,11 \%$ \\
\hline Autónoma Barcelona & 16 & $9,58 \%$ \\
\hline Málaga & 10 & $5,99 \%$ \\
\hline Murcia & 9 & $5,39 \%$ \\
\hline San Pablo-CEU & 7 & $4,19 \%$ \\
\hline País Vasco & 6 & $3,59 \%$ \\
\hline Sevilla & 6 & $3,59 \%$ \\
\hline Extremadura & 5 & $2,99 \%$ \\
\hline Navarra & 5 & $2,99 \%$ \\
\hline Santiago de Compostela & 5 & $2,99 \%$ \\
\hline La Laguna & 3 & $1,79 \%$ \\
\hline Pontificia de Salamanca & 3 & $1,79 \%$ \\
\hline Católica San Antonio & 2 & $1,2 \%$ \\
\hline IES University & 2 & $1,2 \%$ \\
\hline Jaume I Castellón & 2 & $1,2 \%$ \\
\hline Pompeu Fabra & 2 & $1,2 \%$ \\
\hline Ramón Llull & 2 & $1,2 \%$ \\
\hline Rey Juan Carlos & 2 & $1,2 \%$ \\
\hline Valencia & 2 & $1,2 \%$ \\
\hline Alicante & 1 & $0,6 \%$ \\
\hline Cardenal Herrera-CEU & 1 & $0,6 \%$ \\
\hline Granada & 1 & $0,6 \%$ \\
\hline Internacional Cataluña & 1 & $0,6 \%$ \\
\hline Miguel Hernández Elche & 1 & $0,6 \%$ \\
\hline Salamanca & 1 & $0,6 \%$ \\
\hline
\end{tabular}

Tabla 2: Número y porcentaje de tesis defendidas por universidades

\subsection{Dirección de tesis}

Un total de 141 directores han dirigido las 167 tesis analizadas. 107 de ellos (esto es, el 75,9\% del total) han dirigido una única tesis, mientras que otros 26 directores $(18,4 \%)$ han dirigido dos tesis. En la Tabla 3 aparecen los profesores que 
han dirigido tres o más tesis. Como puede verse, la mayoría de ellos pertenece a la Facultad de Ciencias de la Información de la Universidad Complutense de Madrid; si bien el profesor que más tesis doctorales sobre Internet ha dirigido hasta la fecha es José Vicente Rodríguez Muñoz, perteneciente a la Facultad de Comunicación y Documentación de la Universidad de Murcia, centro del que ha sido decano durante varios años. Sin duda, este dato explica en buena medida las cifras obtenidas por dicha universidad en el apartado anterior.

También destaca el hecho de que no aparezca en la tabla ningún director de las Universidad Autónoma de Barcelona ni de la de Málaga, a pesar de ser la segunda y tercera universidades más productivas. Esto se debe a que en ambos casos la producción está muy dispersa, al contrario de lo que ocurre en la Universidad de Murcia, en la que el profesor Rodríguez Muñoz concentra buena parte de esa producción.

Por otro lado, 24 de las tesis $(14,4 \%)$ han sido codirigidas, mientras que las 143 restantes $(85,6 \%)$ contaron con un único director. En ninguno de los casos hubo tres o más directores.

\begin{tabular}{|l|l|l|}
\hline Director & $\mathbf{N}^{\mathbf{0}}$ tesis & Universidad \\
\hline Rodríguez Muñoz, José Vicente & 6 & Murcia \\
\hline Fernández del Moral, Javier & 5 & Complutense \\
\hline García García, Francisco & 5 & Complutense \\
\hline Lallana García, Fernando & 4 & Complutense \\
\hline Cebrián Herreros, Mariano & 3 & Complutense / Sevilla \\
\hline García Alonso Montoya, Pedro & 3 & Complutense \\
\hline García Matilla, Agustín & 3 & Complutense \\
\hline Miguel Arruti, Alberto & 3 & Complutense / San Pablo-CEU \\
\hline
\end{tabular}

Tabla 3: Directores con tres o más tesis dirigidas

\subsection{Presencia en Tribunales y Presidencias}

Lo primero que hay que aclarar en este apartado es que no se ha podido recuperar la información completa relativa la composición de los tribunales de las tesis analizadas, ya que en TESEO faltaban los nombres de los secretarios de cuatro de ellas, y los de dos de los vocales en otras ocho tesis, es decir, en total, han quedado sin identificar 20 identidades de miembros de los tribunales.

El número de académicos presentes en al menos en algún tribunal asciende a 519 , de los que $150(28,9 \%)$ han formado parte de dos o más tribunales y los 369 $(71,1 \%)$ restantes han estado en un único tribunal. En la Tabla 4 se muestra a los profesores que han estado presentes en al menos cinco tribunales o que han presidido al menos tres de ellos. 


\begin{tabular}{|l|l|c|c|}
\hline Nombre & Universidad & Presencias & Presidencias \\
\hline Esteve Ramírez, Francisco & Complutense de Madrid & 13 & 4 \\
\hline Fernández Del Moral, Javier & Complutense de Madrid & 11 & 10 \\
\hline Benavides Delgado, Juan & Complutense de Madrid & 8 & 6 \\
\hline Cebrián Herreros, Mariano & Complutense de Madrid & 7 & 7 \\
\hline $\begin{array}{l}\text { Población Bernardo, José } \\
\text { Ignacio }\end{array}$ & Complutense de Madrid & 7 & 4 \\
\hline $\begin{array}{l}\text { Alvarez Monzoncillo, José } \\
\text { María }\end{array}$ & Rey Juan Carlos & 6 & 1 \\
\hline $\begin{array}{l}\text { Díaz Noci, Javier } \\
\text { Pablos Coello, José }\end{array}$ & $\begin{array}{l}\text { País Vasco / Pompeu } \\
\text { Manuel }\end{array}$ & 6 & 0 \\
\hline $\begin{array}{l}\text { García Fernández, Emilio } \\
\text { Carlos }\end{array}$ & Complutense de Madrid & 6 & 6 \\
\hline Moreno Espinoza, Pastora & Sevilla & 6 & 0 \\
\hline Pérez Amat García, Ricardo & Rey Juan Carlos & 6 & 0 \\
\hline $\begin{array}{l}\text { Cabrera González, Ma } \\
\text { Ángeles }\end{array}$ & Málaga & 5 & 0 \\
\hline Codina Bonilla, Luis & Pompeu Fabra & 5 & 2 \\
\hline Chaparro Escudero, Manuel & Málaga & 5 & 0 \\
\hline Farias García, Pedro & Complutense de Madrid & 5 & 5 \\
\hline Nó Sánchez, Javier & Pontificia Salamanca & 5 & 1 \\
\hline García García, Francisco & Complutense de Madrid & 4 & 3 \\
\hline $\begin{array}{l}\text { Moreiro González, José } \\
\text { Antonio }\end{array}$ & Carlos III & 4 & 4 \\
\hline Bouza Álvarez, Fermín & Complutense de Madrid & 3 & 3 \\
\hline $\begin{array}{l}\text { González } \\
\text { Teodoro }\end{array}$ & Complutense de Madrid & 3 & 3 \\
\hline
\end{tabular}

Tabla 4. Académicos con al menos cinco presencias o tres presidencias en tribunales

Los resultados reflejan que la mayoría de académicos más solicitados pertenecen a la Universidad Complutense de Madrid. A la cabeza de todos ellos, el profesor Fernando Esteve Ramírez, con 13 presencias, seguido de Javier Fernández del Moral con 11 y Juan Benavides Delgado con 8. Les siguen Mariano Cebrián Herreros y José Ignacio Población Bernardo, con 7 presencias cada uno. El primer profesor no perteneciente a la Complutense que aparece en la lista es José María Álvarez Monzoncillo, de la Universidad Rey Juan Carlos, con 6 presencias.

Destaca también la presencia de algunos profesores cuyas universidades no cuentan con una gran producción de tesis doctorales en este campo concreto, como se ha visto anteriormente. Es el caso del mencionado Álvarez Monzoncillo, pero 
también del fallecido profesor de la Universidad Rey Juan Carlos, Ricardo Pérez Amat García; de José Manuel de Pablos Coello (La Laguna); de Luis Codina Bonilla (Pompeu Fabra), de Javier Nó Sánchez (Pontificia de Salamanca) o de José Antonio González (Carlos III). Cabe deducir que, a pesar de esa menor producción de sus respectivas universidades, su prestigio individual les ha llevado a ser requeridos por otras universidades para formar parte de distintos tribunales.

\section{DISCUSIÓN Y CONCLUSIONES}

La tendencia en la producción de tesis sobre Internet muestra alguna que otra diferencia significativa con las reflejadas en otros estudios similares referidos al ámbito de la Comunicación (Marcos Recio, Martínez Pestaña y Blasco López, 2012; Martínez Pestaña, 2011a y 2011b). Televisión (Repiso, Torres y Delgado, 2011; Repiso, Torres y Delgado, 2012; Repiso, Delgado y Torres, 2004; Repiso, Torres-Salina y Delgado López-Cózar, 2013). Todos estos trabajos, ninguno de los cuales analiza más allá del año 2010, coinciden en mostrar un incremento lineal y constante en la producción, con un aumento especialmente notorio entre 2005 y el final del periodo analizado en cada uno de los casos.

Esta tendencia coincide con la observada por Fernández Quijada y Masip (2013) en su estudio sobre las principales revistas académicas españolas de Comunicación. Sus conclusiones hablan de un progresivo incremento del volumen de producción, que se hace especialmente significativo en el último lustro (20052010).

Pues bien, los datos obtenidos en el presente estudio, y en particular los referidos a los años 2004-2007, que muestran como ya se ha comentado un decrecimiento constante en la producción, podrían apuntar a que el proceso de consolidación de Internet como objeto de estudio por parte de los académicos españoles pertenecientes al ámbito de la Comunicación no ha sido una tarea sencilla, y que tras la expectación inicial, con un número de tesis en aumento constante desde la lectura del primer trabajo de este tipo en 1997, ha pasado por un periodo de cierto escepticismo, hasta volver a consolidarse en los últimos años.

Los datos relativos al idioma de lectura coinciden con los obtenidos en otros trabajos precedentes que también han analizado esta categoría (Repiso, Delgado y Torres, 2012; Martínez Pestaña, 2011a y 2011b). Del mismo modo, la presencia testimonial de tesis en idiomas como el inglés o el francés también era testimonial en algunos de esos estudios (Repiso, Delgado y Torres, 2012; Landa Montenegro, 2004).

Lo mismo sucede en el mencionado análisis de Fernández Quijada y Masip (2013), en el que únicamente un 1,2\% de los artículos correspondían a lenguas no oficiales en España. Y algo parecido ocurre en el estudio de Escribà y Cortiñas (2013) también referido a las revistas de Comunicación de nuestro país, pero centrado, entre otros los aspectos, en la internacionalización, y que sitúa en el 7,7\% el porcentaje de artículos que provienen de países no hispanohablantes. 
Por tanto, la presente investigación ratifica la conclusión de estos dos trabajos: que el grado de internacionalización en el ámbito de la Comunicación en España dista todavía mucho de alcanzar los niveles deseados, un hecho común a la producción de artículos y a la de tesis doctorales.

Por otro lado, los hombres representan el $53,9 \%$ de las autorías de las tesis producidas, frente al $46,1 \%$ de las mujeres. Este porcentaje de autoras supone un ligero incremento respecto a otros estudios similares, en los que los hombres tenían una presencia porcentual mayor (Castillo y Xifra, 2006; Repiso, Delgado y Torres, 2012). La presencia mayoritaria de autores en los primeros años y la tendencia creciente en las cifras de autoras también es una tendencia común en estos trabajos.

La producción por universidades está liderada por la Universidad de Complutense, algo que ha sido una constante en muchos estudios similares a éste (Castillo y Xifra, 2006; Marcos Recio, Martínez Pestaña y Blasco López, 2012; Martínez Pestaña, 2004, 2011a y 2011b; Repiso, Torres y Delgado, 2011 y 2012; Repiso, Delgado y Torres, 2012). También aparecen en los primeros puestos de las distintas tablas elaboradas por los autores de estos trabajos otros de los centros con mayor tradición en nuestro país en lo que a estudios de Comunicación se refiere, como la Universidad Autónoma de Barcelona o la de Navarra, e igualmente ocupan lugares destacados en varios casos la Universidad de Málaga (Castillo y Xifra, 2006; Marcos Recio, Martínez Pestaña y Blasco López, 2012; Martínez Pestaña, 2011b; Repiso, Torres y Delgado, 2011) y la de Sevilla (Martínez Pestaña, 2004 y 2011a; Repiso, Torres y Delgado, 2011 y 2012; Repiso, Delgado y Torres, 2012). Sin embargo, en ninguna de estas investigaciones aparecían en lugares tan destacados la Universidad de Murcia ni la de Extremadura, dos centros que en su relativamente corta trayectoria parecen haber prestado especial atención a Internet como objeto de estudio.

Entre los directores más productivos, destaca la figura de José Vicente Rodríguez Muñoz, profesor de la Universidad de Murcia que lidera la tabla y que supone la excepción a la tendencia dominante: la mayoría de directores con mayor producción pertenecen a la Universidad Complutense: es el caso de Javier Fernández del Moral y Francisco García García (cinco tesis cada uno) y de Fernando Lallana García (cuatro). Algunos de estos directores también aparecían entre los más prolíficos en otros estudios similares a éste. Así, el recientemente fallecido profesor Mariano Cebrián Herreros, y los profesores Javier Fernández del Moral, Agustín García Matilla y Francisco García son algunos de los que concentran una mayor producción de tesis sobre televisión (Repiso, Torres y Delgado, 2011), al tiempo que Cebrián Herreros también está entre los directores más prolíficos de tesis sobre radio (Repiso, Torres y Delgado, 2012) y García García entre quienes han dirigido tesis sobre publicidad Marcos Recio, Martínez Pestaña y Blasco López, 2012).

Por otro lado, la codirección es todavía un fenómeno minoritario $(14,4 \%$ del total). Asimismo, entre los profesores que han codirigido alguna de las tesis doctorales analizadas se encuentran varios de los que cuentan con mayor 
producción: José Vicente Rodríguez Muñoz (dos codirecciones), Mariano Cebrián Herreros (una) y Francisco García García (una).

Mientras, los profesores más solicitados para formar parte de tribunales y para presidirlos han sido Fernando Esteve Ramírez, Javier Fernández del Moral y Juan Benavides Delgado. Comparando esta lista con la de los directores más prolíficos, se observan algunas coincidencias, como la del propio Fernández del Moral, o las de Francisco García García o Mariano Cebrián Herreros; pero también la ausencia de algunos de los directores que más tesis sobre Internet han dirigido. Destacan especialmente el caso de José Vicente Rodríguez Muñoz, el académico con un mayor número de tesis dirigidas.

Del mismo modo, al igual que ocurría con las direcciones, algunos de los profesores que han formado parte de un mayor número de tribunales también aparecían en este apartado en otros estudios similares a éste. Así sucede en el caso de Javier Fernández del Moral, en el caso de las tesis sobre televisión (Repiso, Torres y Delgado, 2011) y radio (Repiso, Torres y Delgado, 2012); o Francisco García García en las tesis sobre televisión (Repiso, Torres y Delgado, 2011) y cine (Repiso, Delgado y Torres, 2012). Asimismo, el profesor Pedro Farias García ha sido uno de los más solicitados para formar parte de tesis sobre radio (Repiso, Torres y Delgado, 2012); y los profesores Juan Benavides Delgado y José Manuel De Pablos Coello lo han sido para tesis sobre televisión (Repiso, Torres y Delgado, 2011).

\section{REFERENCIAS BIBLIOGRÁFICAS}

ARBOLEDAS MÁRQUEZ, Luis y HERRERO SOLANA, Víctor (2011). "Una aproximación temática a la producción andaluza en comunicación: análisis a partir de las tesis doctorales de las Universidades de Sevilla y de Málaga". Hipertext.net, 9, Disponible en: http://www.upf.edu/ hipertextnet/numero9/produccion-andaluza-comunicacion.html [Consulta 15/09/2013].

CASANUEVA, Cristóbal y CARO, Francisco Javier (2013). "La Academia Española de Comunicación: productividad científica frente a actividad social". Comunicar, 41, 61-70. (DOI: 10.3916/C41-2013-06).

CASTILLO, Antonio y XIFRA, Jordi (2006). "Investigación bibliométrica de las tesis doctorales españolas sobre relaciones públicas". Anàlisi: quaderns de comunicació i cultura, 34, 141-161.

ESCRIBÀ, Eudald y CORTIÑAS, Sergi (2013). "La internacionalización y las coautorías en las principales revistas científicas de Comunicación en España". Comunicar, 41, 35-44. (DOI: 10.3916/C41-2013-03).

FERNÁNDEZ, David y MASIP, Pere (2013). "Tres décadas de investigación española en comunicación: hacia la mayoría de edad". Comunicar, 41, 15-24. (DOI: 10.3916/C41-2013-01).

FUENTES, Eulalia y ARGUIMBAU, Llorenç (2010). "Las tesis doctorales en España (1997/2008): análisis, estadísticas y repositorios cooperativos". Revista 
Española de Documentación Científica, 33 (1), 63-89. (DOI: 10.3989/redc.2010.1.711)

JONES, Daniel E. et al. (2000). Investigación sobre comunicación en España. Aproximación bibliométrica a las tesis doctorales (1926-1998). Barcelona: ComCat.

JONES, Daniel E. y BARÓ I QUERALT, Jaume (1997). "Tesis doctorals i treballs de recerca universitaris sobre comunicació als Països Catalans 1954-1996: aproximació bibliométrica". Anàlisi: quaderns de comunicació i cultura, 20, 157-187.

LANDA MONTENEGRO, Carmelo (2004). "Recopilación de tesis doctorales sobre Comunicación en las Universidades del País Vasco (1996-2000)". Mediatika, 10, 267-295.

MARCOS RECIO, Juan Carlos; MARTÍNEZ PESTAÑA, María Jesús y BLASCO LÓPEZ, María Francisca (2012). "Producción y dirección de tesis doctorales sobre publicidad en la universidad española (1971-2010)". Revista Española de Documentación Científica, 35 (3), 433-452. (DOI: 10.3989/redc.2012.3.890)

MARTÍNEZ PESTAÑA, María Jesús (2004). "La producción de tesis doctorales sobre temas publicitarios (1971-2001)". Documentación de las Ciencias de la Información, 27, 237-267.

MARTÍNEZ PESTAÑA, María Jesús (2011a). Evaluación de la producción científica sobre publicidad (1971-2001) Tesis doctoral. Facultad de Ciencias de la Información, Universidad Complutense de Madrid.

MARTÍNEZ PESTAÑA, María Jesús (2011b). "La investigación universitaria en Publicidad: producción y temática de las tesis doctorales (1971-2001)". Documentación de las Ciencias de la Información, 34, 119-156.

REPISO, Rafael; TORRES, Daniel y DELGADO, Emilio (2011). "Análisis bibliométrico y de redes sociales en tesis doctorales españolas sobre televisión (1976/2007)". Comunicar, 37, 151-159.

REPISO, Rafael; TORRES, Daniel y DELGADO, Emilio (2012). “Análisis de la investigación sobre radio en España: una aproximación a través del análisis bibliométrico y de redes sociales de las tesis doctorales defendidas en España entre 1976-2008". Estudios sobre el Mensaje Periodístico, 17 (2), 417-429.

REPISO, Rafael; DELGADO, Emilio y TORRES, Daniel (2012). "Análisis bibliométrico de la producción española de Tesis Doctorales sobre Cine 19782007”. En Bort Gual, I., García Catalán, S. y Martín Núñez, M. (eds.). Actas del IV Congreso Internacional Sobre Análisis Fílmico: Nuevas Tendencias e Hibridaciones de los Discursos Audiovisuales en la Cultura Digital Contemporánea. Madrid: Ediciones de las Ciencias Sociales, 976-987.

REPISO, Rafael; TORRES-SALINAS, Daniel y DELGADO LÓPEZ-CÓZAR, Emilio (2013). "La investigación científica sobre Cine en España a partir de sus tesis doctorales: Análisis de redes sociales (1978-2007)". Icono 14, 11 (2), 385404. Disponible en: http://www.icono14.net/ojs/index.php/icono14/article/view/ 530. [Consulta 16/09/2013]. 
SORLI, Ángela y MERLO, José Antonio (2002). "Bases de datos y recursos en Internet de tesis doctorales". Revista Española de Documentación Científica, 25, (1), 95-106. (DOI: 10.3989/redc.2002.v25.i1)

XIFRA, Jordi y CASTILLO, Antonio (2006). "Forty years of doctoral public relations research in Spain: A quantitative study of dissertation contribution to theory development". Public Relations Review, 32 (3), 302-308. 